\title{
Test for interlayer coherence in a quasi-two-dimensional superconductor.
}

\author{
John Singleton ${ }^{1,2}$, P.A. Goddard ${ }^{2}$, A. Ardavan $^{2}$, N. Harrison ${ }^{1}$, S.J. Blundell ${ }^{2}$, J.A. Schlueter ${ }^{3}$ and A.M. Kini ${ }^{3}$ \\ ${ }^{1}$ National High Magnetic Field Laboratory, LANL, \\ MS-E536, Los Alamos, New Mexico 87545, USA \\ ${ }^{2}$ Department of Physics, University of Oxford, Clarendon Laboratory, \\ Parks Road, Oxford OX1 3PU, United Kingdom \\ ${ }^{3}$ Materials Science Division, Argonne National Laboratory, Argonne, Illinois 60439, USA
}

\begin{abstract}
Peaks in the magnetoresistivity of the layered superconductor $\kappa$-(BEDT-TTF $)_{2} \mathrm{Cu}(\mathrm{NCS})_{2}$, measured in fields $\leq 45 \mathrm{~T}$ applied within the layers, show that the Fermi surface is extended in the interlayer direction and enable the interlayer transfer integral $\left(t_{\perp} \approx 0.04 \mathrm{meV}\right)$ to be deduced. However, the quasiparticle scattering rate $\tau^{-1}$ is such that $\hbar / \tau \sim 6 t_{\perp}$, implying that $\kappa$-(BEDT$\mathrm{TTF})_{2} \mathrm{Cu}(\mathrm{NCS})_{2}$ meets the criterion used to identify interlayer incoherence. The applicability of this criterion to anisotropic materials is thus shown to be questionable.

PACS numbers: 74.70.Kn, 78.20.Ls, 71.20.Rv
\end{abstract}

Many correlated-electron systems which are of fundamental interest have very anisotropic electronic bandstructure. Examples include the "high- $T_{\mathrm{c}}$ " cuprates [1, 21, layered ruthenates [3], and crystalline organic metals [2, 1. Such systems may be described by a tightbinding Hamiltonian in which the ratio of the interlayer transfer integral $t_{\perp}$ to the average intralayer transfer integral $t_{||}$is $\ll 1$ [2, 田司. The inequality $\hbar / \tau>t_{\perp}$ [6] where $\tau^{-1}$ is the quasiparticle scattering rate [1, 2, 5], frequently applies to such systems, suggesting that the quasiparticles scatter more frequently than they tunnel between layers. The question has thus arisen as to whether the interlayer charge transfer is coherent or incoherent, i.e. whether or not the Fermi surface (FS) extends in the interlayer direction [2, 4, 5. In this paper we have used magnetoresistance data to estimate the interlayer transfer integral in the highly anisotropic organic superconductor $\kappa$-(BEDT-TTF $)_{2} \mathrm{Cu}(\mathrm{NCS})_{2}$. We find that the material obeys the inequality $\hbar / \tau>t_{\perp}$; moreover, mean-free path in the interlayer direction is $\lesssim 20 \%$ of the unit-cell height. Nevertheless, our data demonstrate a FS which is extended in the interlayer direction.

$\kappa$-(BEDT-TTF $)_{2} \mathrm{Cu}(\mathrm{NCS})_{2}$ was selected for our experiments because it is perhaps the most thoroughly characterised quasi-two-dimensional (Q2D) conductor 化. In contrast to the cuprates, the FS topology is well known from Shubnikov-de Haas (SdH) and de Haas-van Alphen

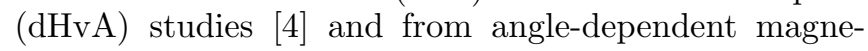
toresistance oscillation (AMRO) [8] and millimetre-wave (MMW) experiments [9]; it consists of a pair of quasione-dimensional (Q1D) electron sheets plus a Q2D hole pocket (see Fig. 11a 10, 11]). The $\kappa$-phase BEDT-TTF salts are considered to be leading contenders for interlayer incoherence [5], and optical data may be interpreted as consistent with this suggestion [12]. Moreover, models for unconventional superconductivity in $\kappa$ phase BEDT-TTF salts invoke the nesting properties of the FS [11, 13, 14]; the degree of nesting might depend on whether the FS is a 2D or 3D entity (see [ 4 , Section 3.5). Experimental tests for coherence in $\kappa$ (BEDT-TTF $)_{2} \mathrm{Cu}(\mathrm{NCS})_{2}$ are thus far deemed to be in- conclusive [5]; e.g. semiclassical models can reproduce AMRO [8] and MMW data [9] equally well when the interlayer transport is coherent or "weakly coherent" [0].

To examine how interlayer coherence might be detected, we use a tight-binding dispersion relationship $-2 t_{\perp} \cos \left(k_{\perp} a\right)$ to represent the interlayer dispersion, where $k_{\perp}$ is the interlayer component of $\mathbf{k}$ and $a$ is the interlayer spacing. This is added to the effective dimer model, which is known to represent the intralayer bandstructure accurately 10, 11, to yield

$$
\begin{aligned}
E(\mathbf{k})= & \pm 2 \cos \left(\frac{k_{\mathbf{b}} b}{2}\right) \sqrt{t_{\mathbf{c} 1}^{2}+t_{\mathbf{c} 2}^{2}+2 t_{\mathbf{c} 1} t_{\mathbf{c} 2} \cos \left(k_{\mathbf{c}} c\right)} \\
& +2 t_{\mathbf{b}} \cos \left(k_{\mathbf{b}} b\right)-2 t_{\perp} \cos \left(k_{\perp} a\right) .
\end{aligned}
$$

Here $k_{\mathbf{b}}$ and $k_{\mathbf{c}}$ are the intralayer components of $\mathbf{k}$ (see Fig. 1 $1 \mathrm{a})$ and $t_{\mathbf{b}}, t_{\mathbf{c} 1}$ and $t_{\mathbf{c} 2}$ are interdimer transfer integrals 10, 11]; the + and - signs result in the Q1D sheets and Q2D pocket of the FS respectively (Fig. 11a). The addition of the interlayer dispersion produces a warping of the FS, shown schematically for the Q2D section in Fig. 1 $1 \mathrm{~b}$; the FS cross-section is modulated in the interlayer direction. This modulation might suggest that separate "neck and belly" frequencies would be observed in the dHvA effect [4]; however, only a single frequency is seen in low-field studies [16], suggesting that the cyclotron energy exceeds $t_{\perp}$ at the fields at which quantum oscillations are observed.

Having failed to detect the beating between neck and bellies, and in view of the inconclusive nature of other tests [5], we have chosen instead to examine the behaviour of $\kappa$-(BEDT-TTF $)_{2} \mathrm{Cu}(\mathrm{NCS})_{2}$ in almost exactly in-plane magnetic fields [5, 17, 18]. The motion of quasiparticles of charge $q$ in a magnetic field $\mathbf{B}$ is determined by the Lorentz force $\hbar \dot{\mathbf{k}}=q \mathbf{v} \times \mathbf{B}$, where the quasiparticle velocity is given by $\hbar \mathbf{v}=\nabla_{\mathbf{k}} E$ [19, 20]; this leads to orbits on the FS in planes perpendicular to $\mathbf{B}$. Hence, if the FS is extended in the interlayer direction, an in-plane field will cause closed orbits on the bellies (see Fig. 1 b). Such orbits are effective at averaging $v_{\perp}$, 
the interlayer component of the velocity, and their presence will lead to an increase in the magnetoresistivity component $\rho_{z z} 117,18,19$. B can then be tilted away from the in-plane direction by an angle $\Delta$, such that the small closed orbits about the bellies cease to be possible; this occurs when $\mathbf{B}$ is parallel to $\mathbf{v}$ at the point at which $v_{\perp}$ is a maximum (i.e. when the normal to the FS is at a maximum angle to the Q2D planes). Therefore, on tilting $\mathbf{B}$ around the in-plane orientation, we expect to see a peak in $\rho_{z z}$, of angular width $2 \Delta$, if (and only if [5]) the FS is extended in the interlayer direction.

A problem in using $\kappa$-(BEDT-TTF $)_{2} \mathrm{Cu}(\mathrm{NCS})_{2}$ is its high in-plane critical field; $\mu_{0} H_{\mathrm{c} 2}(T=0) \approx 35 \mathrm{~T}$, falling to $\mu_{0} H_{\mathrm{c} 2} \approx 25 \mathrm{~T}$ at $4.2 \mathrm{~K}$ [21]. Moreover, apparent peaks in the resistivity of $\kappa$-(BEDT-TTF $)_{2} \mathrm{Cu}(\mathrm{NCS})_{2}$ in in-plane fields may occur due to dissipative processes associated with vortices within the mixed state [4, 22]. To ensure that such effects do not interfere with our data, we choose to stay at fields well above the superconducting state; our measurements were therefore carried out in the $45 \mathrm{~T}$ hybrid magnet at NHMFL Tallahassee. The experiments involved two single crystals $\left(\sim 0.7 \times 0.5 \times 0.1 \mathrm{~mm}^{3}\right.$; mosaic spread $\left.\lesssim 0.1^{\circ}\right)$ of $\kappa$-(BEDT-TTF $)_{2} \mathrm{Cu}(\mathrm{NCS})_{2}$, produced using electrocrystallization [15]. In one of the crystals, the terminal hydrogens of the BEDT-TTF molecules were isotopically substituted by deuterium; we refer to the deuterated sample as $\mathrm{d} 8$, and the hydrogenated sample as h8 23. Both crystals were mounted in a ${ }^{3} \mathrm{He}$ cryostat which allowed rotation to all possible orientations in B [21]; sample orientation is defined by the angle $\theta$ between B and the normal to the sample's Q2D planes and the azimuthal angle $\phi(\phi=0$ is a plane of rotation of $\mathbf{B}$ containing $\mathbf{k}_{\mathbf{b}}$ and the normal to the Q2D planes). The interlayer magnetoresistance $R_{z z}\left(\propto \rho_{z z}\right)$ was measured using the four-terminal ac techniques described in [21].

Fig. 2 shows $R_{z z}$ of the d8 sample close to the inplane orientation $\theta=90^{\circ}$; data for three values of $\phi$ are shown. (The data for the h8 sample were very similar in all respects [23.) The edges of Fig. 2 are dominated by AMROs and related phenomena; as these are well known [5, 19, 25] in $\kappa$-(BEDT-TTF $)_{2} \mathrm{Cu}(\mathrm{NCS})_{2}$ [8], we shall not describe them further in this paper. Close to $\theta=90^{\circ}$, there is a distinct peak in $R_{z z}$, the width and height of which vary with $\phi$; we attribute this peak to the closed orbits described above.

Fig. 3 shows the peak at temperatures $T$ from $0.48 \mathrm{~K}$ to $5.1 \mathrm{~K}$; increasing $T$ by over an order of magnitude reduces the peak height but has little effect on its width 24. Varying the field (in the range $35-45 \mathrm{~T}$ at $0.5 \mathrm{~K}$, and in the range $29-45 \mathrm{~T}$ at $4.2 \mathrm{~K}$ ) also has little effect on the peak width; increases of field result in increases in peak height and definition, in a manner similar to the field dependence of AMROs [19]. Both of these observations support the idea that the peak is a consequence of the FS geometry alone.

Fig. 1 shows the variation of $2 \Delta$, the full width of the peak close to $\theta=90^{\circ}$ versus $\phi$; the width was deduced using the extrapolations shown Fig. 3 (inset). In order to interpret this variation, we use Eqn. 11 to calculate $v_{\perp \max }$, the maximum value of $v_{\perp}$, and $v_{\|}$, the intralayer velocity component parallel to the plane of rotation of $\mathbf{B}$; when measured in radians, $\Delta \approx v_{\perp \max } / v_{\|}$. As all of the relevant quasiparticle motion occurs close to the Fermi energy, $E \approx E_{\mathrm{F}}$, we adjust the parameters of Eqn. 1 to reproduce the known FS parameters of $\kappa$ $(\mathrm{BEDT}-\mathrm{TTF})_{2} \mathrm{Cu}(\mathrm{NCS})_{2}$. First, $t_{\mathbf{c}} / t_{\mathbf{b}}$ (where $t_{\mathbf{c}}$ is the mean of $t_{\mathbf{c} 1}$ and $\left.t_{\mathbf{c} 2}\right)$ is adjusted to obtain the dHvA frequencies of the Q2D pocket and the magnetic breakdown orbit [10, 23, 26]. The absolute value of $t_{\mathbf{c}}$ is then constrained by fitting to the effective mass of the breakdown orbit [4, 23]. Third, the energy gap measured in magnetic breakdown $\left(E_{\mathrm{g}} \approx 7.8 \mathrm{meV}\right.$ [26]) gives $t_{\mathbf{c} 1}-t_{\mathbf{c} 2}=$ $E_{\mathrm{g}} / 2$ [10], leading to $t_{\mathbf{b}}=15.6 \mathrm{meV}, t_{\mathbf{c} 1}=24.2 \mathrm{meV}$ and $t_{\mathbf{c} 2}=20.3 \mathrm{meV}$. Finally, $a=16.2 \AA[15]$, so that equations for $\Delta$ contain only one adjustable parameter, $t_{\perp}$. The substitution of $t_{\perp}=0.04 \mathrm{meV}$ leads to the curves shown in Fig. A; the continuous curve is from the Q2D FS, and the loops are caused by the Q1D sheets, which support closed orbits only over a restricted range of $\phi$. In the latter case, the lower branch of the loop results from the flatter portions of the Q1D FS, roughly parallel to $\mathbf{k}_{\mathbf{c}}$, whereas the upper branch is caused by the more highly curved region at the zone boundary (Fig. 11a).

For most $\phi$, there is agreement between curve and data (Fig. 4), but around $\phi=0$ and $180^{\circ}$ it seems that the observed peak width is sometimes dominated by closed orbits on the Q1D sheets, and sometimes by those on the Q2D FS section; the dominant width presumably depends on which FS section is more effective at averaging $v_{\perp}$ [19]. MMW studies [9] suggest that the interlayer corrugations of the Q1D sheets are more complex than those given by Eqn. 11. This could lead to a rapid variation with $\phi$ of the effectiveness of the orbits in increasing $\rho_{z z}$. For a narrow range of angles close to $\phi=25^{\circ}$, the peak was particularly wide and large (see top trace, Fig. 2). This is perhaps connected to the gap between the Q1D and Q2D FS sections.

As mentioned above, the bandstructure of $\kappa$-(BEDT$\mathrm{TTF})_{2} \mathrm{Cu}(\mathrm{NCS})_{2}$ within the Q2D layers is determined by the interdimer transfer integrals [10, 11]. A better guide to the total intralayer bandwidth than the parameters used above (which are relevant for $E \approx E_{\mathrm{F}}$ and thus include renormalising interactions [4, 10]) is given by the fits to optical data of Ref. [10], which suggest $t_{\mathbf{b}} \approx 60 \mathrm{meV}$ and $t_{\mathbf{c}} \approx 120 \mathrm{meV}$; these are a factor $\gtrsim 10^{3}$ larger than $t_{\perp} \approx 0.04 \mathrm{meV}$.

The failure of the dHvA effect [16] to observe necks and bellies may now be understood; the Landau-level spacing at the lowest $|\mathbf{B}|$ used $(\sim 6 \mathrm{~T})$ is $0.2 \mathrm{meV}, \sim 5 t_{\perp}$.

It is instructive to compare $t_{\perp}$ with $\tau^{-1}$. Samples $\mathrm{d} 8$ and h8 have been studied using $\mathrm{SdH}$ oscillations at $|\mathbf{B}| \leq 15 \mathrm{~T}[25]$. At such fields, the oscillatory magnetoresistance is much less than the nonoscillatory component, and magnetic breakdown is a minor consideration [4]. Hence, the 2D form of the Lifshitz-Kosevich formula may be used to extract $\tau$ [4], giving $\tau=2.9 \pm 0.5 \mathrm{ps}(\mathrm{h} 8)$ and 
$\tau=2.6 \pm 0.3 \mathrm{ps}$ (d8) [25]. Another estimate can be derived from MMW studies [9], which measure the FStraversal resonance (FTR) due to quasiparticles crossing the Q1D FS sheets; these experiments used samples from the same batch as h8. In the data of [9], the FTR appears at $B \approx 10 \mathrm{~T}$, with a full-width at half-maximum of $\Delta B \approx 7 \mathrm{~T}$. If we assume that $\omega \tau \sim B / \Delta B$ [27], where $\omega=2 \pi \times 70 \times 10^{9} \mathrm{rad} \mathrm{s}^{-1}$ [9], we obtain $\tau \sim 3 \mathrm{ps}$, close to the $\mathrm{SdH}$ values. Thus, $\hbar / \tau \approx 0.24 \mathrm{meV}, \sim 6 t_{\perp}$.

Finally, we estimate the intralayer mean-free path $\lambda_{\|}$; a typical value of $v_{\|}\left(\approx 7.2 \times 10^{4} \mathrm{~ms}^{-1}\right)$, yields $\lambda_{\|}=v_{\|} \tau \approx 0.2 \mu \mathrm{m}$ (i.e. $\kappa$-(BEDT-TTF $)_{2} \mathrm{Cu}(\mathrm{NCS})_{2}$ is a very clean system). However, the maximum interlayer velocity is $v_{\perp \max }=2 t_{\perp} a / \hbar \approx 200 \mathrm{~ms}^{-1}$, suggesting an average interlayer velocity $\overline{v_{\perp}} \sim 100 \mathrm{~ms}^{-1}$, and a meanfree path $\lambda_{\perp} \sim \bar{v}_{\perp} \tau \approx 3 \AA$. The usual mean-free path formalism implies that only $\sim \exp (-16.2 / 3) \approx 0.5 \%$ of the quasiparticles travels between adjacent layers (separation $16.2 \AA$ [15]) without scattering. In such circumstances, conventional criteria [5] lead one to expect incoherent interlayer transport, yet the peak in $\rho_{z z}$ described above unambiguously demonstrates a 3D FS topology.

The criterion for incoherent transport was developed for isotropic disordered metals [7]; the mean free path $\lambda$ was taken to represent the spatial extent over which the Bloch waves are coherent and is, by default, isotropic. However, in Q2D systems $\lambda_{\|} \gg \lambda_{\perp}$, so that a purely ballistic model of transport (of the sort used to treat disorded 3D systems [7]) fails to preserve information on the spatial extent of the wavefunctions perpendicular to the layers. Our experiment therefore demonstrates serious inadequacies in such models. When impurities and defects are randomly dispersed throughout a material, the inter-impurity separation should be almost isotropic. Hence, we should also expect the spatial coherence of the Bloch states to remain roughly isotropic, even when the group velocity is highly anisotropic.

In summary, we observe a peak in the interlayer resistance of the highly anisotropic superconductor $\kappa$-(BEDT$\mathrm{TTF})_{2} \mathrm{Cu}(\mathrm{NCS})_{2}$ when a magnetic field is applied within the layers. This demonstrates [5] that the Fermi surface is extended in the interlayer direction, and allows the interlayer transfer integral to be estimated to be $t_{\perp} \approx$ $0.04 \mathrm{meV}\left(t_{\perp} / k_{\mathrm{B}} \approx 0.5 \mathrm{~K}\right)$. The small size of $t_{\perp}$ explains unsuccessful attempts to observe neck and belly orbits in dHvA studies. However, perhaps the most interesting point to emerge is that $\kappa$-(BEDT-TTF $)_{2} \mathrm{Cu}(\mathrm{NCS})_{2}$ obeys the criteria commonly used to delineate interlayer incoherence - the interlayer mean free path is smaller than the unit cell size, and $\hbar / \tau \geq t_{\perp}$ - and yet it clearly possesses a three-dimensional Fermi surface. It is perhaps now time to re-examine the validity of such criteria when applied to strongly anisotropic systems.

This work is supported by EPSRC (UK). NHMFL is supported by the US Department of Energy (DoE), the National Science Foundation and the State of Florida. Work at Argonne is sponsored by the DoE, Office of Basic Energy Sciences, Division of Materials Science under contract number W-31-109-ENG-38. We thank Vic Emery and Jane Symington for stimulating discussions.
[1] L.B. Ioffe and A.J. Millis, Science 285, 1241 (2000).

[2] D.G. Clarke and S.P. Strong, Adv. Phys. 46, 545 (1997)

[3] C. Bergemann et al., Phys. Rev. Lett. 84, 2662 (2000).

[4] J. Singleton, Rep. Prog. Phys. 63, 1111 (2000).

[5] R.H. McKenzie and P. Moses, Phys. Rev. Lett.81, 4492 (1998); Phys. Rev. B 60, 11241 (1999).

[6] This is roughly equivalent to the Mott-Ioffe-Regel criterion [7]; see e.g. [5] or Section 7.2 of [2].

[7] N.F. Mott and E.H. Davies Electronic properties of noncrystalline materials (Taylor and Francis, London, 1975); A.F. Ioffe and A.R. Regel, Prog. Semicond., 4, 237 (1960).

[8] M.S. Nam et al., Synth. Met. 103, 1905 (1999).

[9] J.M. Schrama et al., J. Phys.: Condens. Matter 13, 2235 (2001).

[10] J.M. Caulfield et al., J. Phys.: Condens. Matter 6, 2911 (1994).

[11] Jörg Schmalian, Phys. Rev. Lett. 81, 4232 (1998).

[12] J.J. McGuire et al., preprint cond-mat 0103237.

[13] R. Louati et al. Synth. Met. 103, 1857 (1999).

[14] K. Kuroki and H. Aoki, Phys. Rev. B 603060 (1999).

[15] H. Uryama et al., Chem. Lett. 1988, 463 (1988).

[16] T. Sasaki et al., Phys. Rev. B. 57, 10889 (1998).

[17] T. Osada et al., Phys. Rev. Lett. 77, 5261 (1996); N. Hanasaki et al., Phys. Rev. B 57, 1336 (1998); ibid. 60, 11210 (1999)

[18] Others propose that "self-crossing orbits" (SCOs) are more effective than closed orbits in averaging $v_{\perp}$. However, the geometrical constraints on the peak in $\rho_{z z}$ are identical for closed orbits and SCOs if the in-plane dispersion relationship is parabolic (see V.G. Peschansky and M.V. Kartsovnik, Phys. Rev. B 60, 11207 (1999); I.J. Lee and M.J. Naughton, Phys. Rev. B 57, 7423 (1998)). It is straightforward to show that the bands predicted by Equation 1 are almost parabolic at $E \approx E_{\mathrm{F}}$.

[19] M.S. Nam et al., J. Phys.: Condens. Matter 13, 2271 (2001).

[20] N.W. Ashcroft and N.D. Mermin, Solid State Physics, Saunders (1976).

[21] J. Singleton et al. J. Phys.: Condens. Matter 12, L641 (2000).

[22] M. Chaparala et al., Phys. Rev. B 53, 5818 (1996).

[23] AMRO and SdH data [25] show that $\mathrm{d} 8$ and h8 have very similar FSs; e.g. the $\mathrm{SdH}$ frequency and effective mass of the Q2D pocket in $\mathrm{d} 8(\mathrm{~h} 8)$ are $597 \pm 1 \mathrm{~T}(600 \pm 1 \mathrm{~T})$ and $3.4 \pm 0.1 m_{\mathrm{e}}\left(3.5 \pm 0.1 m_{\mathrm{e}}\right) 25$. The only marked difference is that the magnetic breakdown probability is somewhat higher in $\mathrm{d} 8$ 25].

[24] This observation also calls into question the assertion that interlayer transport is incoherent if $k_{\mathrm{B}} T>t_{\perp}$ (see e.g. P. Anderson, The theory of superconductivity in the high High $T_{\mathrm{c}}$ cuprates, Princeton University Press, 1997, $\mathrm{p} 50)$.

[25] P.A. Goddard et al., to be published. 
(a)

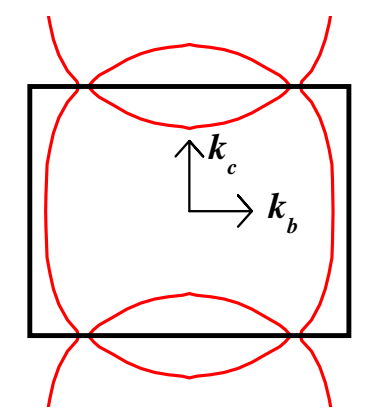

(b)

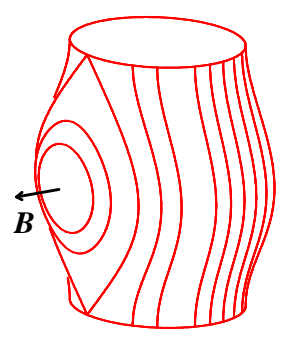

[26] N. Harrison et al., J. Phys.: Condens. Matter, 8, 5415 (1996).

[27] e.g. B.I. and B. Bleaney, Electricity and Magnetism, (Oxford University Press, 1990) p. 734.

FIG. 1: (a) Cross-section of the Fermi surface (FS) and Brillouin zone of $\kappa$-(BEDT-TTF $)_{2} \mathrm{Cu}(\mathrm{NCS})_{2}$ predicted by Eqn. 1 [10, 11]. (b) Perspective view of the Q2D FS section described by Eqn 11; the intralayer curvature and interplane warping have been exaggerated for clarity. The lines indicate quasiparticle orbits on the FS due to the in-plane field $\mathbf{B}$. Note the closed orbits about the "belly" of the FS. 


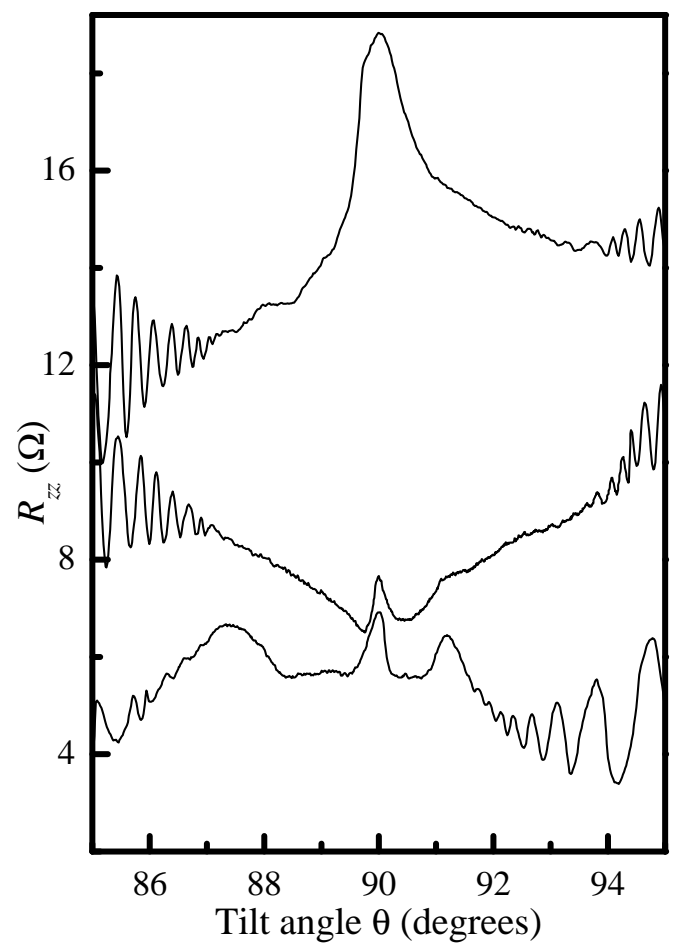

FIG. 2: Interlayer resistance $R_{z z}$ for the $\mathrm{d} 8 \kappa$-(BEDT$\mathrm{TTF})_{2} \mathrm{Cu}(\mathrm{NCS})_{2}$ sample as a function of tilt angle $\theta$. Data for three planes of rotation of the field are shown, $\phi=25^{\circ}$ (upper), $\phi=20^{\circ}$ (middle) and $\phi=15^{\circ}$ (lower). The static magnetic field is $42 \mathrm{~T}$, and the temperature is $520 \mathrm{mK}$. 


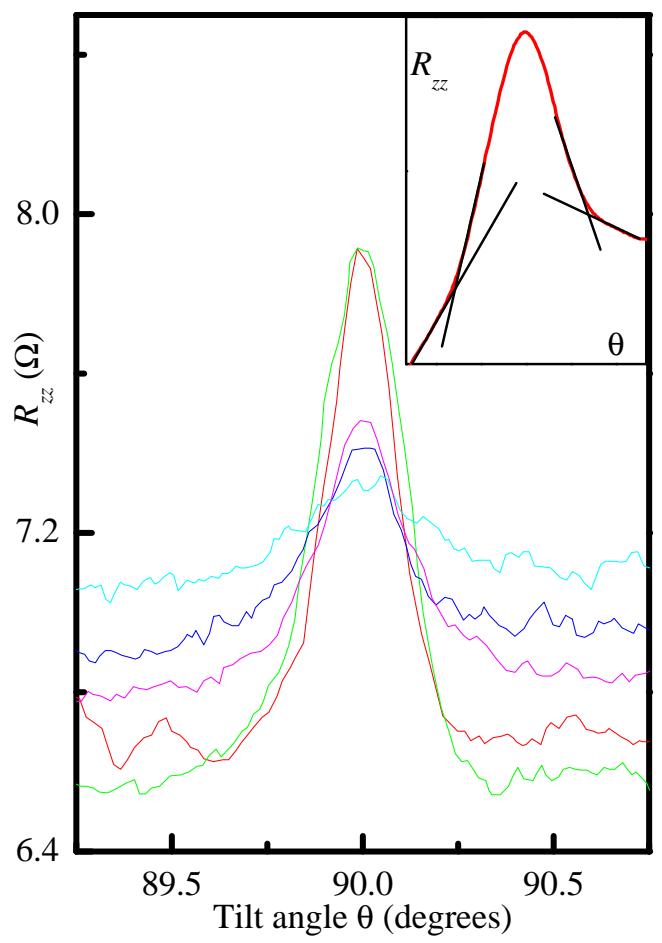

FIG. 3: Interlayer resistance $\left(R_{z z}\right)$ versus angle $\theta$ for temperatures $T=0.48 \mathrm{~K}, 1.4 \mathrm{~K}, 3.0 \mathrm{~K}, 4.4 \mathrm{~K}$ and $5.1 \mathrm{~K}\left(\phi=135^{\circ}\right)$. The background magnetoresistance increases with increasing $T$, whereas the peak at $\theta=90^{\circ}$ becomes smaller. The data shown are for the $\mathrm{d} 8 \kappa$-(BEDT-TTF $)_{2} \mathrm{Cu}(\mathrm{NCS})_{2}$ sample. The inset shows the intersections of the linear extrapolations used to determine the peak width. 


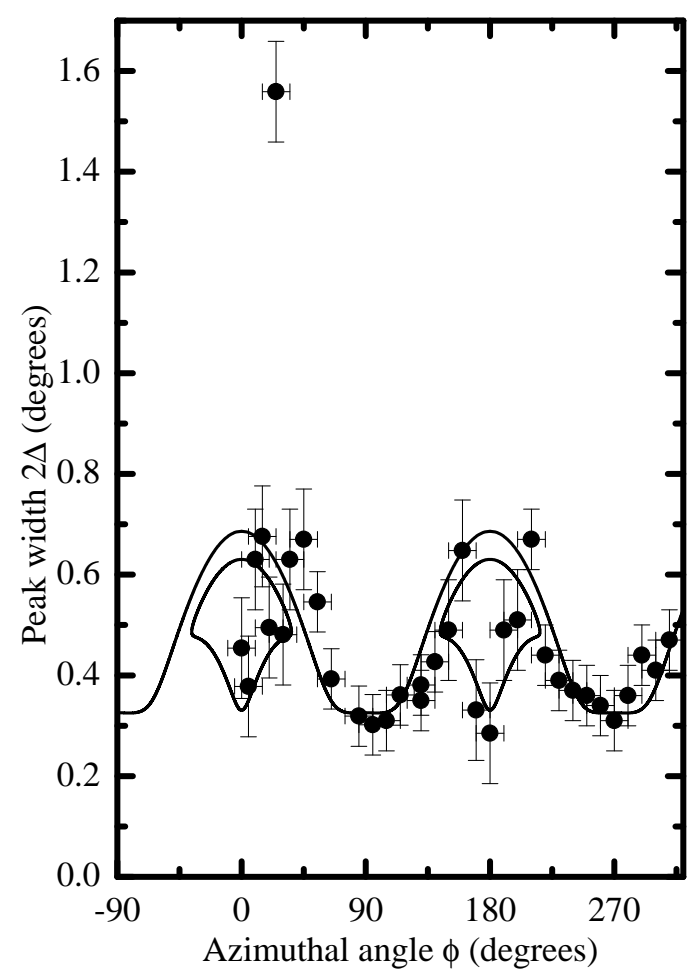

FIG. 4: Angular width $2 \Delta$ of peak in $R_{z z}(B=42 \mathrm{~T}, T \approx$ $500 \mathrm{mK}$ ) versus azimuthal angle $\phi$; data for the $\mathrm{d} 8 \kappa$-(BEDT$\mathrm{TTF})_{2} \mathrm{Cu}(\mathrm{NCS})_{2}$ sample are shown. Points are data; the curves represent the model prediction with $t_{\perp}=0.04 \mathrm{meV}$. The continuous curve is due to the Q2D FS section; the Q1D sheets can only support closed orbits over a restricted range of $\phi$, leading to the top-shaped loops. 